Bull. Chem. Soc. Ethiop. 2015, 29(3), 457-471.

Printed in Ethiopia

DOI: http://dx.doi.org/10.4314/bcse.v29i3.14

ISSN 1011-3924

(c) 2015 Chemical Society of Ethiopia

\title{
SUCCINIMIDE- $N$-SULFONIC ACID AS AN EFFICIENT RECYCLABLE CATALYST FOR THE SYNTHESIS OF SOME FUSED INDOLO PYRANO PYRIMIDINONE DERIVATIVES
}

\author{
S. Sheik Mansoor ${ }^{*}$, K. Logaiya, S.P.N. Sudhan and K. Aswin \\ Research Department of Chemistry, Bioactive Organic Molecule Synthetic Unit, \\ C. Abdul Hakeem College (Autonomous), Melvisharam - 632 509, Tamil Nadu, India
}

(Received August 29, 2014; revised June 8, 2015)

\begin{abstract}
A new, simple, thermally efficient and solvent-free condensation of 2-amino-4,5-dihydro-4phenylpyrano[3,2-b]indole-3-carbonitrile derivatives with coumarin-3-carboxylic acid employing succinimide- $N$ sulfonic acid (SuSA) as catalyst for the synthesis of a series of 5,6-dihydro-2-(2-oxo-2H-chromen-3-yl)-5-phenylindolo[2',3':5,6]pyrano[2,3- $d]$ pyrimidin-4(3H)-one derivatives is described. This method has the advantages of high yield, simple methodology, and short reaction time, as well as being green in terms of avoiding the use of toxic catalysts and solvents. Furthermore, the catalyst could be recycled and reused four times without significant loss of activity. Thiourea dioxide (TUD) catalyzed efficient three-component coupling reactions of aromatic aldehydes, 3-hydroxyindole and malononitrile in water at $70{ }^{\circ} \mathrm{C}$ was described as the preparation of 2-amino-4,5dihydro-4-phenylpyrano[3,2-b]indole-3-carbonitrile derivatives.
\end{abstract}

KEY WORDS: Succinimide- $N$-sulfonic acid, Thiourea dioxide, Coumarin-3-carboxylic acid, Indolo pyrano pyrimidineone derivatives

\section{INTRODUCTION}

Coumarins are secondary heterocyclic metabolites composed of fused benzene and $\alpha$-pyrone rings, and they occur widely in different parts of plants, such as roots, seeds, nuts, flowers and fruits [1]. The pharmacological and biochemical properties and therapeutic applications of coumarins depend upon the pattern of substitution and have attracted intense interest in recent years because of their diverse pharmacological properties [2]. The coumarin derivatives have demonstrated significant potential for use in a wide range of biological applications such as antioxidant [3], anticancer [4, 5], anti-proliferative [6], anti-tuberculosis [7] and antimicrobial activities [8]. Some novel coumarin-3-carboxamide derivatives linked to $N$-benzylpiperidine scaffold were synthesized and evaluated as acetylcholinesterase (AChE) and butyrylcholinesterase (BuChE) inhibitors [9]. Osteoporosis is a progressive skeletal disorder, due to the unequal coupling between osteoclast mediated bone resorption and osteoblast mediated bone formation [10, 11]. Anti-osteoporotic effects of the newly synthesized coumarine pyridine hybrids were evaluated in primary cultures of rat calvarial osteoblasts in vitro [12]. The observed interesting biological properties of this class of compounds impelled us to synthesize new examples.

In view of the pharmaceutical importance of heterocyclic compounds containing coumarin moiety, various approaches toward the synthesis of this class of compounds have been explored [13-17]. Although these methods are quite satisfactory, most of these methods suffer from extended reaction times, low yields, use of costly reagents, vigorous reaction conditions and also requirement of tedious work-up procedures. Therefore, development of a simple, efficient, inexpensive and environment friendly process for the synthesis of coumarins is highly desirable.

The principles of green chemistry have been introduced to eliminate or reduce the use of hazardous materials [18]. Solvent-free organic reactions have attracted much interest particularly from the viewpoint of green chemistry. Implementation of organic transformations under solvent-free reaction conditions have gained in popularity in recent years because of their

*Corresponding author. E-mail: smansoors2000@yahoo.co.in 
simple workup procedure, high efficiency, mild conditions, environmental friendliness, cleanliness, low cost, handling, and economical friendliness [19, 20]. In addition the growing concern for the influence of the chemical reagents on the environment as well as on human body, recovery and reusability of the chemical reagents has attracted the attention of synthetic organic chemists. More importantly pharmaceutical industry has given more importance towards recovery and reuse of chemical reagents to reduce the cost of a product as well as the environmental burden.

Recently, succinimide- $N$-sulfonic acid (SuSA) has been used as a catalyst for chemoselective trimethylsilylation of alcohols and phenols with hexamethyldisilazane (HMDS) [21], chemoselective conversion of amines to their corresponding $N$-Boc protected derivatives with (Boc) $)_{2} \mathrm{O}$ [22] and also for the acetylation reactions in the absence of a solvent [23]. As part of continuing effort in our laboratory towards the development of environmentally friendly procedures for the synthesis of biologically active heterocyclic molecules [24-26], we now describe the synthesis of 5,6-dihydro-2-(2-oxo-2H-chromen-3-yl)-5-phenyl-indolo[2',3':5,6]pyrano[2,3-d]pyrimidin-4(3H)-one derivatives from the reaction of 2-amino-4,5-dihydro-4phenylpyrano[3,2-b]indole-3-carbonitriles and coumarine-3-carboxylic acid using SuSA as an efficient reusable catalyst under solvent-free conditions. 2-Amino-4,5-dihydro-4-phenylpyrano[3,2-b]indole-3-carbonitriles were synthesized from benzaldehydes, 3-hydroxyindole and malononitrile using TUD as an efficient organocatalyst (Scheme 1).<smiles>[R]c1ccc(C=O)cc1</smiles>

1a-j<smiles>Oc1c[nH]c2ccccc12</smiles>

2<smiles>NC(N)=S(=O)=O</smiles>

$(0.1 \mathrm{mmol})$<smiles></smiles><smiles>N#CC1=C(N)Oc2c([nH]c3ccccc23)C1c1ccccc1</smiles>

$4 a-j$

Scheme 1. Synthesis of various 2-amino-4,5-dihydro-4-phenylpyrano[3,2-b]indole-3carbonitrile derivatives from benzaldehyde, 3-hydroxyindole and malononitrile catalysed by TUD $(10 \mathrm{~mol} \%)$ in water at $70^{\circ} \mathrm{C}$.

In recent years, TUD is used as the catalyst for the hydrolysis of imines [27], synthesis of naphthopyran derivatives [28], synthesis of a library of novel heterocyclic compounds [29], synthesis of 3,4-dihydropyrimidinones [30] and for the catalytic oxidation of alcohols [31]. We have also reported the application of TUD for the synthesis of 3,4-dihydropyrano[c]chromenes and 6-amino-5-cyano-4-aryl-2-methyl-4H-pyrans in water [26]. Multicomponent reactions (MCRs) allow the creation of several bonds in a single operation and are attracting increasing attention as one of the most powerful emerging synthetic tools for the creation of molecular diversity and complexity [32-34].

\section{EXPERIMENTAL}

\section{Apparatus and analysis}

Chemicals were purchased from Merck, Fluka and Aldrich Chemical Companies. All yields refer to isolated products unless otherwise stated. ${ }^{1} \mathrm{H}$ NMR $(500 \mathrm{MHz})$ and ${ }^{13} \mathrm{C}$ NMR $(125 \mathrm{MHz})$ spectra were obtained using Bruker DRX-500 Avance at ambient temperature, using TMS as internal standard. FT-IR spectra were obtained as $\mathrm{KBr}$ discs on Shimadzu spectrometer. Mass 
spectra were determined on a Varion - Saturn 2000 GC/MS instrument. Elemental analysis was measured by means of Perkin Elmer $2400 \mathrm{CHN}$ elemental analyzer flowchart.

Preparation of succinimide- $N$-sulfonic acid

Succinimide- $N$-sulfonic acid as a stable reagent was easily prepared by the reaction of succinimide with neat chlorosulfonic acid (Scheme 2) [21].

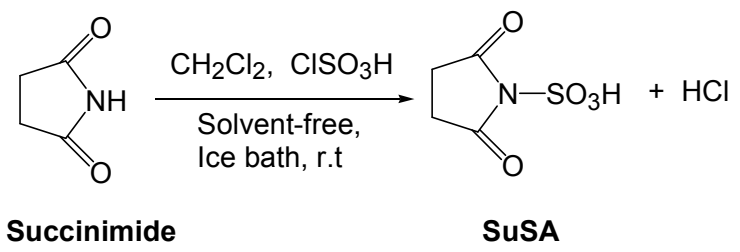

Scheme 2. Preparation of SuSA.

General procedure for the synthesis of pyrano[3,2-b] indole derivatives (4a-j)

A mixture of aldehyde (1 mmol), 3-hydroxyindole $(1 \mathrm{mmol})$, malononitrile $(1.1 \mathrm{mmol})$ and TUD $(0.1 \mathrm{mmol})$ in water $(5 \mathrm{~mL})$ was stirred at $70{ }^{\circ} \mathrm{C}$ (Scheme 1). After completion of the reaction, as indicated by TLC, ethanol $(10 \mathrm{~mL})$ was added and the reaction mixture was filtered. The remaining solution was washed with warm ethanol $(3 \times 5 \mathrm{~mL})$ in order to separate organocatalyst. After cooling, the crude products were precipitated. The remaining aqueous thiourea dioxide was collected and reused without any further processing for subsequent runs. The reaction products were identified by comparing their physical and spectral data (i.e., IR, ${ }^{1} \mathrm{H}$ and ${ }^{13} \mathrm{C}$ NMR and MS) with those reported in the literature for the same compounds. The crude products were purified by recrystallization from ethanol (95\%) to give $\mathbf{4 a - j}$.

Spectral date for the synthesized pyranoindole derivatives

2-Amino-4,5-dihydro-4-phenylpyrano[3,2-b]indole-3-carbonitrile (4a). IR $\left(\mathrm{KBr}, \mathrm{cm}^{-1}\right)$ : 3276 and $3241\left(-\mathrm{NH}_{2}\right), 2213(-\mathrm{CN}), 1660(-\mathrm{NH}) ;{ }^{1} \mathrm{H}$ NMR $\left(500 \mathrm{MHz}, \mathrm{CDCl}_{3}\right) \delta: 5.22(\mathrm{~s}, 1 \mathrm{H}, \mathrm{CH})$, 6.88 (bs, 2H, NH $), 6.77-6.90(\mathrm{~m}, 2 \mathrm{H}, \mathrm{Ar}-\mathrm{H}) ; 7.04-7.13(\mathrm{~m}, 5 \mathrm{H}, \mathrm{Ar}-\mathrm{H}) ; 7.52(\mathrm{~d}, 1 \mathrm{H}, J=7.4$, Ar-H); 7.77 (d, 1H, $J=7.8$, Ar-H), $9.80(\mathrm{bs}, 1 \mathrm{H}, \mathrm{NH}) \mathrm{ppm} ;{ }^{13} \mathrm{C}$ NMR $\left(125 \mathrm{MHz}, \mathrm{CDCl}_{3}\right) \delta$ : $30.2,59.8,103.1,111.3,117.4,120.5,122.1,128.0,128.9,130.2,130.8,135.0,135.5,137.4$, 177.0 ppm; MS(ESI): $m / z 288(\mathrm{M}+\mathrm{H})^{+}$. Anal. calcd for $\mathrm{C}_{18} \mathrm{H}_{13} \mathrm{~N}_{3} \mathrm{O}: \mathrm{C}, 75.25 ; \mathrm{H}, 4.56 ; \mathrm{N}$, $14.63 \%$. Found: C, 75.13; H, 4.52; N, 14.60\%.

2-Amino-4,5-dihydro-4-(4-fluorophenyl)-pyrano[3,2-b]indole-3-carbonitrile (4b). IR ( $\mathrm{KBr}$, $\left.\mathrm{cm}^{-1}\right): 3270$ and $3234\left(-\mathrm{NH}_{2}\right), 2221(-\mathrm{CN}), 1664(-\mathrm{NH}) ;{ }^{1} \mathrm{H}$ NMR $\left(500 \mathrm{MHz}, \mathrm{CDCl}_{3}\right) \delta: 5.15$ (s, 1H, CH), 6.93 (bs, 2H, NH $), 6.80-6.97$ (m, 2H, Ar-H); 7.04-7.13 (m, 4H, Ar-H); 7.48 (d, $1 \mathrm{H}, J=7.4, \mathrm{Ar}-\mathrm{H}) ; 7.85(\mathrm{~d}, 1 \mathrm{H}, J=7.8, \mathrm{Ar}-\mathrm{H}), 9.94(\mathrm{bs}, 1 \mathrm{H}, \mathrm{NH}) \mathrm{ppm} ;{ }^{13} \mathrm{C}$ NMR $(125 \mathrm{MHz}$, $\left.\mathrm{CDCl}_{3}\right) \delta: 30.5,60.3,102.9,110.8,118.2,121.0,122.9,127.6,128.7,130.6,131.1,135.3$, 135.7, 138.0, 176.4 ppm; MS(ESI): $m / z 306(\mathrm{M}+\mathrm{H})^{+}$. Anal. calcd for $\mathrm{C}_{18} \mathrm{H}_{12} \mathrm{FN}_{3} \mathrm{O}: \mathrm{C}, 70.82 ; \mathrm{H}$, $3.93 ; \mathrm{N}, 13.77 \%$. Found: C, 70.75; H, 3.90; N, 13.75\%.

2-Amino-4,5-dihydro-4-(3-bromophenyl)-pyrano[3,2-b]indole-3-carbonitrile (4c). IR ( $\mathrm{KBr}$, $\left.\mathrm{cm}^{-1}\right): 3268$ and $3245\left(-\mathrm{NH}_{2}\right), 2208(-\mathrm{CN}), 1656(-\mathrm{NH}) ;{ }^{1} \mathrm{H}$ NMR $\left(500 \mathrm{MHz}, \mathrm{CDCl}_{3}\right) \delta: 5.24(\mathrm{~s}$, $1 \mathrm{H}, \mathrm{CH}), 6.78$ (bs, 2H, NH$)_{2}$, 6.74-6.89 (m, 2H, Ar-H); 7.14-7.23 (m, 4H, Ar-H); 7.58 (d, 1H, $J$ 
$=7.4, \mathrm{Ar}-\mathrm{H}) ; 7.76(\mathrm{~d}, 1 \mathrm{H}, J=7.8, \mathrm{Ar}-\mathrm{H}), 9.95(\mathrm{bs}, 1 \mathrm{H}, \mathrm{NH}) \mathrm{ppm} ;{ }^{13} \mathrm{C} \mathrm{NMR}\left(125 \mathrm{MHz}, \mathrm{CDCl}_{3}\right)$ $\delta: 30.0,59.5,102.7,110.6,117.6,120.6,122.2$, 128.1, 128.6, 130.1 130.9, 135.6, 135.9, 137.6, $177.1 \mathrm{ppm}$; MS(ESI): $\mathrm{m} / z 366.9(\mathrm{M}+\mathrm{H})^{+}$. Anal. calcd for $\mathrm{C}_{18} \mathrm{H}_{12} \mathrm{BrN}_{3} \mathrm{O}: \mathrm{C}, 59.03 ; \mathrm{H}, 3.28 ; \mathrm{N}$, $11.48 \%$. Found: C, 58.91; H, 3.22; N, 11.44\%.

2-Amino-4,5-dihydro-4-(2-chlorophenyl)-pyrano[3,2-b]indole-3-carbonitrile (4d). IR ( $\mathrm{KBr}$, $\left.\mathrm{cm}^{-1}\right): 3266$ and $3233\left(-\mathrm{NH}_{2}\right), 2219(-\mathrm{CN}), 1669(-\mathrm{NH}) ;{ }^{1} \mathrm{H} \mathrm{NMR}\left(500 \mathrm{MHz}, \mathrm{CDCl}_{3}\right) \delta: 5.17(\mathrm{~s}$, $1 \mathrm{H}, \mathrm{CH}), 6.90$ (bs, 2H, NH$)_{2}, 6.84-6.97$ (m, 2H, Ar-H); 7.09-7.19 (m, 4H, Ar-H); 7.45 (d, 1H, $J$ $=7.4, \mathrm{Ar}-\mathrm{H}) ; 7.90(\mathrm{~d}, 1 \mathrm{H}, J=7.8, \mathrm{Ar}-\mathrm{H}), 9.78(\mathrm{bs}, 1 \mathrm{H}, \mathrm{NH}) \mathrm{ppm} ;{ }^{13} \mathrm{C} \mathrm{NMR}\left(125 \mathrm{MHz}, \mathrm{CDCl}_{3}\right)$ $\delta: 30.9,60.7,103.0,111.2,118.1,121.2,122.7,127.5,128.5,130.0,131.2,135.1,135.5,138.1$, $176.7 \mathrm{ppm}$; MS(ESI): $\mathrm{m} / z 322.5(\mathrm{M}+\mathrm{H})^{+}$. Anal. calcd for $\mathrm{C}_{18} \mathrm{H}_{12} \mathrm{ClN}_{3} \mathrm{O}: \mathrm{C}, 67.19 ; \mathrm{H}, 3.73 ; \mathrm{N}$, $13.06 \%$. Found: C, 67.10; H, 3.75; N, 13.01\%.

2-Amino-4,5-dihydro-4-(4-cyanophenyl)-pyrano[3,2-b]indole-3-carbonitrile (4e). IR $(\mathrm{KBr}$, $\left.\mathrm{cm}^{-1}\right): 3270$ and $3238\left(-\mathrm{NH}_{2}\right), 2216(-\mathrm{CN}), 1672(-\mathrm{NH}) ;{ }^{1} \mathrm{H}$ NMR $\left(500 \mathrm{MHz}, \mathrm{CDCl}_{3}\right) \delta: 5.14(\mathrm{~s}$, $1 \mathrm{H}, \mathrm{CH}), 6.85$ (bs, 2H, NH$\left.)_{2}\right), 6.76-6.94(\mathrm{~m}, 2 \mathrm{H}, \mathrm{Ar}-\mathrm{H}) ; 7.17-7.33(\mathrm{~m}, 4 \mathrm{H}, \mathrm{Ar}-\mathrm{H}) ; 7.56(\mathrm{~d}, 1 \mathrm{H}, J$ $=7.4, \mathrm{Ar}-\mathrm{H}) ; 7.84(\mathrm{~d}, 1 \mathrm{H}, J=7.8, \mathrm{Ar}-\mathrm{H}), 9.74(\mathrm{bs}, 1 \mathrm{H}, \mathrm{NH}) \mathrm{ppm} ;{ }^{13} \mathrm{C} \mathrm{NMR}\left(125 \mathrm{MHz}, \mathrm{CDCl}_{3}\right)$ $\delta: 31.4,59.6,102.6,110.9,116.8,117.7,120.8,122.0,128.3,128.8,130.4$ 130.8, 135.4, 135.7, 137.6, 177.3 ppm; MS(ESI): $m / z 313(\mathrm{M}+\mathrm{H})^{+}$. Anal. calcd for $\mathrm{C}_{19} \mathrm{H}_{12} \mathrm{~N}_{4} \mathrm{O}: \mathrm{C}, 73.07 ; \mathrm{H}, 3.85 ; \mathrm{N}$, $17.95 \%$. Found: C, $73.01 ; \mathrm{H}, 3.83 ; \mathrm{N}, 17.83 \%$.

2-Amino-4,5-dihydro-4-(4- $\mathrm{N}, \mathrm{N}$-dimethylaminophenly)-pyrano[3,2-b] indole-3-carbonitrile (4f). IR $\left(\mathrm{KBr}, \mathrm{cm}^{-1}\right): 3274$ and $3240\left(-\mathrm{NH}_{2}\right), 2206(-\mathrm{CN}), 1659(-\mathrm{NH}) ;{ }^{1} \mathrm{H}$ NMR $\left(500 \mathrm{MHz}, \mathrm{CDCl}_{3}\right)$ $\delta: 2.72\left(\mathrm{~s}, 6 \mathrm{H}, \mathrm{N}\left(\mathrm{CH}_{3}\right)_{2}\right), 5.22(\mathrm{~s}, 1 \mathrm{H}, \mathrm{CH}), 6.82\left(\mathrm{bs}, 2 \mathrm{H}, \mathrm{NH}_{2}\right), 6.85-6.94(\mathrm{~m}, 2 \mathrm{H}, \mathrm{Ar}-\mathrm{H}) ; 7.19-$ $7.30(\mathrm{~m}, 4 \mathrm{H}, \mathrm{Ar}-\mathrm{H}) ; 7.54(\mathrm{~d}, 1 \mathrm{H}, J=7.4, \mathrm{Ar}-\mathrm{H}) ; 7.83(\mathrm{~d}, 1 \mathrm{H}, J=7.8, \mathrm{Ar}-\mathrm{H}), 9.83$ (bs, $1 \mathrm{H}, \mathrm{NH})$ ppm; ${ }^{13} \mathrm{C}$ NMR $\left(125 \mathrm{MHz}, \mathrm{CDCl}_{3}\right) \delta: 31.3,42.8,60.5,103.4,111.2,118.0,121.3,122.3,127.7$, 128.4, 130.7, 131.0, 135.7, 136.2, 138.0, $176.6 \mathrm{ppm}$; MS(ESI): $\mathrm{m} / z 331(\mathrm{M}+\mathrm{H})^{+}$. Anal. calcd for $\mathrm{C}_{20} \mathrm{H}_{18} \mathrm{~N}_{4} \mathrm{O}: \mathrm{C}, 72.72 ; \mathrm{H}, 5.45 ; \mathrm{N}, 16.96 \%$. Found: C, 72.60; H, 5.46; N, 16.84\%.

2-Amino-4,5-dihydro-4-(2-nitrophenyl)-pyrano[3,2-b]indole-3-carbonitrile (4g). IR ( $\mathrm{KBr}$, $\left.\mathrm{cm}^{-1}\right): 3271$ and $3243\left(-\mathrm{NH}_{2}\right), 2212(-\mathrm{CN}), 1662(-\mathrm{NH}) ;{ }^{1} \mathrm{H}$ NMR $\left(500 \mathrm{MHz}, \mathrm{CDCl}_{3}\right) \delta: 5.26$ (s, 1H, CH), 6.77 (bs, 2H, NH $), 6.86-6.98$ (m, 2H, Ar-H); 7.12-7.28 (m, 4H, Ar-H); 7.47 (d, $1 \mathrm{H}, J=7.4, \mathrm{Ar}-\mathrm{H}) ; 7.76(\mathrm{~d}, 1 \mathrm{H}, J=7.8, \mathrm{Ar}-\mathrm{H}), 9.87(\mathrm{bs}, 1 \mathrm{H}, \mathrm{NH}) \mathrm{ppm} ;{ }^{13} \mathrm{C}$ NMR $(125 \mathrm{MHz}$, $\left.\mathrm{CDCl}_{3}\right) \delta: 31.5,59.9,102.9,110.7,117.9,120.7,122.7,128.2,128.8,130.3,130.7,135.5$, 136.1, 137.5, 177.0 ppm; MS(ESI): $m / z 333(\mathrm{M}+\mathrm{H})^{+}$. Anal. calcd for $\mathrm{C}_{18} \mathrm{H}_{12} \mathrm{~N}_{4} \mathrm{O}_{3}: \mathrm{C}, 65.06 ; \mathrm{H}$, $3.61 ; \mathrm{N}, 16.87 \%$. Found: C, 64.96; H, 3.57; N, 16.85\%.

2-Amino-4,5-dihydro-4-(3-methylphenyl)-pyrano[3,2-b]indole-3-carbonitrile (4h). IR ( $\mathrm{KBr}$, $\left.\mathrm{cm}^{-1}\right): 3268$ and $3237\left(-\mathrm{NH}_{2}\right), 2217(-\mathrm{CN}), 1670(-\mathrm{NH}) ;{ }^{1} \mathrm{H} \mathrm{NMR}\left(500 \mathrm{MHz}, \mathrm{CDCl}_{3}\right) \delta: 2.16(\mathrm{~s}$, $\left.3 \mathrm{H}, \mathrm{CH}_{3}\right), 5.13(\mathrm{~s}, 1 \mathrm{H}, \mathrm{CH}), 6.95$ (bs, 2H, $\left.\mathrm{NH}_{2}\right), 6.80-6.95(\mathrm{~m}, 2 \mathrm{H}, \mathrm{Ar}-\mathrm{H}) ; 7.08-7.18(\mathrm{~m}, 4 \mathrm{H}$, Ar-H); $7.53(\mathrm{~d}, 1 \mathrm{H}, J=7.4, \mathrm{Ar}-\mathrm{H}) ; 7.91(\mathrm{~d}, 1 \mathrm{H}, J=7.8, \mathrm{Ar}-\mathrm{H}), 9.80$ (bs, $1 \mathrm{H}, \mathrm{NH}) \mathrm{ppm} ;{ }^{13} \mathrm{C}$ NMR $\left(125 \mathrm{MHz}, \mathrm{CDCl}_{3}\right) \delta: 20.1,30.4,60.2,103.0,111.0,118.3,121.1,122.5,127.8,128.7$, 130.2, 131.0, 135.0, 135.7, 138.0, $176.8 \mathrm{ppm} ; \mathrm{MS}(\mathrm{ESI}): \mathrm{m} / \mathrm{z} 302(\mathrm{M}+\mathrm{H})^{+}$. Anal. calcd for $\mathrm{C}_{19} \mathrm{H}_{15} \mathrm{~N}_{3} \mathrm{O}: \mathrm{C}, 75.75 ; \mathrm{H}, 4.98 ; \mathrm{N}, 13.95 \%$. Found: $\mathrm{C}, 75.66 ; \mathrm{H}, 4.99 ; \mathrm{N}, 13.93 \%$.

2-Amino-4,5-dihydro-4-(4-chlorophenyl)-pyrano[3,2-b]indole-3-carbonitrile (4i). IR ( $\mathrm{KBr}$, $\left.\mathrm{cm}^{-1}\right)$ : 3276 and $3236\left(-\mathrm{NH}_{2}\right), 2212(-\mathrm{CN}), 1667(-\mathrm{NH}) ;{ }^{1} \mathrm{H}$ NMR $\left(500 \mathrm{MHz}, \mathrm{CDCl}_{3}\right) \delta: 5.17(\mathrm{~s}$, 1H, CH), 6.79 (bs, 2H, NH$)_{2}$, 6.93-7.02 (m, 2H, Ar-H); 7.08-7.19 (m, 4H, Ar-H); 7.38 (d, 1H, $J$ $=7.4, \mathrm{Ar}-\mathrm{H}) ; 7.83(\mathrm{~d}, 1 \mathrm{H}, J=7.8, \mathrm{Ar}-\mathrm{H}), 9.81(\mathrm{bs}, 1 \mathrm{H}, \mathrm{NH}) \mathrm{ppm} ;{ }^{13} \mathrm{C} \mathrm{NMR}\left(125 \mathrm{MHz}, \mathrm{CDCl}_{3}\right)$ $\delta$ : $30.7,60.7,103.7,111.3,118.3,121.3,122.4,127.5,128.3,130.2,131.2,135.0,135.7,138.4$, 
$176.5 \mathrm{ppm}$; MS(ESI): $m / z 322.5(\mathrm{M}+\mathrm{H})^{+}$. Anal. calcd for $\mathrm{C}_{18} \mathrm{H}_{12} \mathrm{ClN}_{3} \mathrm{O}: \mathrm{C}, 67.19 ; \mathrm{H}, 3.73 ; \mathrm{N}$, $13.06 \%$. Found: C, $67.13 ; \mathrm{H}, 3.67 ; \mathrm{N}, 12.94 \%$.

2-Amino-4,5-dihydro-4-(4-methylphenyl)-pyrano[3,2-b]indole-3-carbonitrile (4j). IR ( $\mathrm{KBr}$, $\left.\mathrm{cm}^{-1}\right): 3266$ and $3236\left(-\mathrm{NH}_{2}\right), 2216(-\mathrm{CN}), 1669(-\mathrm{NH}) ;{ }^{1} \mathrm{H} \mathrm{NMR}\left(500 \mathrm{MHz}, \mathrm{CDCl}_{3}\right) \delta: 2.19(\mathrm{~s}$, $\left.3 \mathrm{H}, \mathrm{CH}_{3}\right), 5.19(\mathrm{~s}, 1 \mathrm{H}, \mathrm{CH}), 6.84\left(\mathrm{bs}, 2 \mathrm{H}, \mathrm{NH}_{2}\right), 6.93-7.07(\mathrm{~m}, 2 \mathrm{H}, \mathrm{Ar}-\mathrm{H}) ; 7.11-7.18(\mathrm{~m}, 4 \mathrm{H}$, $\operatorname{Ar}-\mathrm{H}) ; 7.53(\mathrm{~d}, 1 \mathrm{H}, J=7.4$, Ar-H); $7.88(\mathrm{~d}, 1 \mathrm{H}, J=7.8, \mathrm{Ar}-\mathrm{H}), 9.88$ (bs, $1 \mathrm{H}, \mathrm{NH}) \mathrm{ppm} ;{ }^{13} \mathrm{C}$ NMR $\left(125 \mathrm{MHz}, \mathrm{CDCl}_{3}\right) \delta: 18.7,30.7,61.2,102.2,111.3,117.3,121.2,122.4,127.6,128.5$, 130.2, 131.1, 134.8, 135.3, 138.4, 176.7 ppm; MS(ESI): $m / z 302(\mathrm{M}+\mathrm{H})^{+}$. Anal. calcd for $\mathrm{C}_{19} \mathrm{H}_{15} \mathrm{~N}_{3} \mathrm{O}$ : C, 75.75; H, 4.98; N, 13.95\%. Found: C, 75.68; H, 4.91; N, 13.89\%.

General procedure for the synthesis of 5,6-dihydro-2-(2-oxo-2H-chromen-3-yl)-5-phenylindolo[2',3':5,6]pyrano[2,3-d]pyrimidin-4(3H)-one derivatives by SuSA

A mixture of 2-amino-4,5-dihydro-4-phenylpyrano[3,2-b]indole-3-carbonitrile 4a-j (1 mmol), coumarin-3-carboxylic acid $(1 \mathrm{mmol})$ and SuSA $(0.05 \mathrm{mmol})$ were heated at $80{ }^{\circ} \mathrm{C}$ for about $3.0-4.0 \mathrm{~h}$ (Scheme 3). After completion of the reaction as indicated by TLC, the insoluble crude product was dissolved in hot ethanol and the SuSA was filtered off. The filtrate was concentrated to dryness, and the crude product was purified by recrystallization from ethanol. The recovered catalyst was washed with acetone, dried and reused for subsequent reactions without loss in its activity and product yield.<smiles>[R]c1cccc(C2C(C#N)=C(N)Oc3c2[nH]c2ccccc32)c1</smiles>

$4 a j$

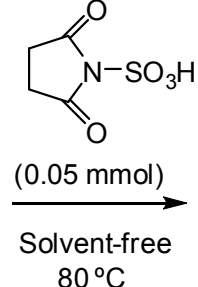

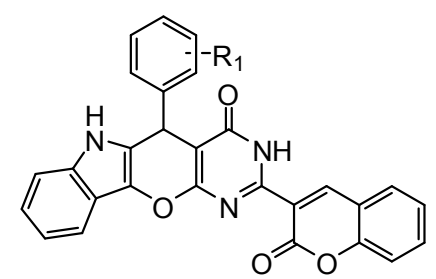

$6 a-j$

Scheme 3. Synthesis of various 5,6-dihydro-2-(2-oxo-2 $H$-chromen-3-yl)-5-phenylindolo[2',3':5,6]pyrano[2,3-d]pyrimidin-4(3H)-one derivatives from $\mathbf{4 a - j}$ and coumarine-3-carboxylic acid catalysed by SuSA $(0.05 \mathrm{mmol})$ under solvent-free condition at $80^{\circ} \mathrm{C}$.

Spectral data for the synthesized compounds (6a-j)

5,6-Dihydro-2-(2-oxo-2H-chromen-3-yl)-5-phenyl-indolo[2',3':5,6]pyrano[2,3-d]pyrimidin4(3H)-one (6a). IR $\left(\mathrm{KBr}, \mathrm{cm}^{-1}\right): 3266,1708,1677,1660,1633,1604,1200 ;{ }^{1} \mathrm{H}$ NMR $(500$ $\mathrm{MHz}_{\mathrm{CDCl}}$ ) $\delta: 4.96(\mathrm{~s}, 1 \mathrm{H}, \mathrm{CH}), 6.82-6.94(\mathrm{~m}, 2 \mathrm{H}, \mathrm{Ar}-\mathrm{H}) ; 7.14-7.28(\mathrm{~m}, 5 \mathrm{H}, \mathrm{Ar}-\mathrm{H}) ; 7.44$ (d, $1 \mathrm{H}, J=7.6, \mathrm{Ar}-\mathrm{H}) ; 7.60(\mathrm{~d}, 1 \mathrm{H}, J=8.0, \mathrm{Ar}-\mathrm{H}), 7.80-8.00(\mathrm{~m}, 4 \mathrm{H}, \mathrm{Ar}-\mathrm{H}), 8.26(\mathrm{~s}, 1 \mathrm{H}$, coumarin $\mathrm{H}), 8.77(\mathrm{~s}, 1 \mathrm{H}, \mathrm{NH}) 9.94(\mathrm{~s}, 1 \mathrm{H}, \mathrm{NH}) \mathrm{ppm} ;{ }^{13} \mathrm{C} \mathrm{NMR}\left(125 \mathrm{MHz}, \mathrm{CDCl}_{3}\right) \delta: 30.8,102.1,103.6$, $111.2,117.7,120.3,121.2,122.5,125.1,125.5,126.2,127.5,128.0,128.2,128.5,129.3,135.6$, $137.3,137.7,146.4,150.7,160.9,162.4,164.1,168.4$ ppm; MS(ESI): $m / z 460(\mathrm{M}+\mathrm{H})^{+}$. Anal. calcd for $\mathrm{C}_{28} \mathrm{H}_{17} \mathrm{~N}_{3} \mathrm{O}_{4}$ : C, 73.20; H, 3.70; N, 9.15\%. Found: C, 73.11; H, 3.66; N, 9.12\%.

5,6-Dihydro-2-(2-oxo-2H-chromen-3-yl)-5-(4-fluorophenyl)-indolo[2',3':5, 6] pyrano[2,3d]pyrimidin-4(3H)-one (6b). IR ( $\left.\mathrm{KBr}, \mathrm{cm}^{-1}\right): 3277,1704,1673,1669,1635,1596,1204 ;{ }^{1} \mathrm{H}$ 
NMR (500 MHz, $\left.\mathrm{CDCl}_{3}\right) \delta: 5.06(\mathrm{~s}, 1 \mathrm{H}, \mathrm{CH}), 6.75-6.96(\mathrm{~m}, 2 \mathrm{H}, \mathrm{Ar}-\mathrm{H}) ; 7.10-7.30(\mathrm{~m}, 4 \mathrm{H}, \mathrm{Ar}-$ $\mathrm{H}) ; 7.46(\mathrm{~d}, 1 \mathrm{H}, J=7.6, \mathrm{Ar}-\mathrm{H}) ; 7.68(\mathrm{~d}, 1 \mathrm{H}, J=8.0, \mathrm{Ar}-\mathrm{H}), 7.78-7.96(\mathrm{~m}, 4 \mathrm{H}, \mathrm{Ar}-\mathrm{H}), 8.30$ (s, $1 \mathrm{H}$, coumarin $\mathrm{H}), 8.86(\mathrm{~s}, 1 \mathrm{H}, \mathrm{NH}) 10.06(\mathrm{~s}, 1 \mathrm{H}, \mathrm{NH}) \mathrm{ppm} ;{ }^{13} \mathrm{C} \mathrm{NMR}\left(125 \mathrm{MHz}, \mathrm{CDCl}_{3}\right) \delta$ : $31.2,102.6,103.3,111.6,117.5,120.2,121.4,122.9,124.7,125.4,126.7,127.3,128.2,128.3$, 128.6, 130.0, 135.0, 137.2, 137.4, 146.3, 151.3, 161.2, $162.1164 .6,168.2 \mathrm{ppm}$; MS(ESI): $\mathrm{m} / \mathrm{z}$ $478(\mathrm{M}+\mathrm{H})^{+}$. Anal. calcd for $\mathrm{C}_{28} \mathrm{H}_{16} \mathrm{FN}_{3} \mathrm{O}_{4}: \mathrm{C}, 70.44 ; \mathrm{H}, 3.35 ; \mathrm{N}, 8.80 \%$. Found: $\mathrm{C}, 70.34 ; \mathrm{H}$, $3.30 ; \mathrm{N}, 8.77 \%$.

5,6-Dihydro-2-(2-oxo-2H-chromen-3-yl)-5-(3-bromophenyl)-indolo[2',3':5, 6]pyrano[2,3d]pyrimidin-4(3H)-one (6c). IR ( $\left.\mathrm{KBr}, \mathrm{cm}^{-1}\right): 3282,1711,1685,1662,1638,1605,1211 ;{ }^{1} \mathrm{H}$ NMR $\left(500 \mathrm{MHz}, \mathrm{CDCl}_{3}\right) \delta: 5.08(\mathrm{~s}, 1 \mathrm{H}, \mathrm{CH}), 6.84-6.92(\mathrm{~m}, 2 \mathrm{H}, \mathrm{Ar}-\mathrm{H}) ; 7.16-7.32(\mathrm{~m}, 4 \mathrm{H}, \mathrm{Ar}-$ $\mathrm{H}) ; 7.50(\mathrm{~d}, 1 \mathrm{H}, J=7.6, \mathrm{Ar}-\mathrm{H}) ; 7.75(\mathrm{~d}, 1 \mathrm{H}, J=8.0, \mathrm{Ar}-\mathrm{H}), 7.85-7.99$ (m, 4H, Ar-H), $8.42(\mathrm{~s}$, $1 \mathrm{H}$, Coumarin $\mathrm{H}), 8.80(\mathrm{~s}, 1 \mathrm{H}, \mathrm{NH}) 9.98(\mathrm{~s}, 1 \mathrm{H}, \mathrm{NH}) \mathrm{ppm} ;{ }^{13} \mathrm{C} \mathrm{NMR}\left(125 \mathrm{MHz}, \mathrm{CDCl}_{3}\right) \delta$ : $30.7,102.7,103.2,110.4,118.0,120.4,121.5,123.2$, 125.7, 125.9, 126.7, 127.7, 128.3, 128.5, $128.7,130.2,135.4,137.7,137.9,147.0,150.6,160.8,162.5,165.0,167.7 \mathrm{ppm} ; \mathrm{MS}(\mathrm{ESI}): \mathrm{m} / \mathrm{z}$ $538.9(\mathrm{M}+\mathrm{H})^{+}$. Anal. calcd for $\mathrm{C}_{28} \mathrm{H}_{16} \mathrm{BrN}_{3} \mathrm{O}_{4}$ : C, 62.46; H, 2.97; N, 7.81\%. Found: $\mathrm{C}, 62.40 ; \mathrm{H}$, $2.94 ; \mathrm{N}, 7.80 \%$.

5,6-Dihydro-2-(2-oxo-2H-chromen-3-yl)-5-(2-chlorophenyl)-indolo[2',3':5,6]pyrano[2,3-

d]pyrimidin-4(3H)-one (6d). IR $\left(\mathrm{KBr}, \mathrm{cm}^{-1}\right)$ : 3270, 1713, 1680, 1670, 1643, 1592, 1213; ${ }^{1} \mathrm{H}$ NMR (500 MHz, $\left.\mathrm{CDCl}_{3}\right) \delta: 4.92(\mathrm{~s}, 1 \mathrm{H}, \mathrm{CH}), 6.78-6.89(\mathrm{~m}, 2 \mathrm{H}, \mathrm{Ar}-\mathrm{H}) ; 7.09-7.29(\mathrm{~m}, 4 \mathrm{H}, \mathrm{Ar}-$ $\mathrm{H}) ; 7.54$ (d, $1 \mathrm{H}, J=7.6$, Ar-H); 7.70 (d, 1H, $J=8.0$, Ar-H), 7.82-8.01 (m, 4H, Ar-H), 8.30 (s, $1 \mathrm{H}$, coumarin $\mathrm{H}), 8.73(\mathrm{~s}, 1 \mathrm{H}, \mathrm{NH}) 10.10(\mathrm{~s}, 1 \mathrm{H}, \mathrm{NH}) \mathrm{ppm} ;{ }^{13} \mathrm{C} \mathrm{NMR}\left(125 \mathrm{MHz}, \mathrm{CDCl}_{3}\right) \delta$ : $31.4,102.0,104.2,111.3,118.3,120.3,121.5,123.4,125.3,125.7,126.3,127.3,128.3,128.6$, $128.8,129.5,135.2,138.1,138.6,147.2,151.0,161.3,162.7,164.7,167.9 \mathrm{ppm} ; \mathrm{MS}(\mathrm{ESI}): \mathrm{m} / \mathrm{z}$ $493.4(\mathrm{M}+\mathrm{H})^{+}$. Anal. calcd for $\mathrm{C}_{28} \mathrm{H}_{16} \mathrm{ClN}_{3} \mathrm{O}_{4}$ : C, 68.09; $\mathrm{H}, 3.24 ; \mathrm{N}, 8.51 \%$. Found: $\mathrm{C}, 68.01 ; \mathrm{H}$, $3.26 ; \mathrm{N}, 8.47 \%$

5,6-Dihydro-2-(2-oxo-2H-chromen-3-yl)-5-(4-cyanophenyl)-indolo[2',3':5,6]pyrano[2,3-

d]pyrimidin-4(3H)-one (6e). IR $\left(\mathrm{KBr}, \mathrm{cm}^{-1}\right): 3262,1707,1683,1668,1637,1607,1202 ;{ }^{1} \mathrm{H}$ NMR $\left(500 \mathrm{MHz}, \mathrm{CDCl}_{3}\right) \delta: 4.99(\mathrm{~s}, 1 \mathrm{H}, \mathrm{CH}), 6.74-6.92(\mathrm{~m}, 2 \mathrm{H}, \mathrm{Ar}-\mathrm{H}) ; 7.18-7.40(\mathrm{~m}, 4 \mathrm{H}, \mathrm{Ar}-$ $\mathrm{H}) ; 7.58(\mathrm{~d}, 1 \mathrm{H}, J=7.6, \mathrm{Ar}-\mathrm{H}) ; 7.69$ (d, $1 \mathrm{H}, J=8.0, \mathrm{Ar}-\mathrm{H}), 7.85-7.94(\mathrm{~m}, 4 \mathrm{H}, \mathrm{Ar}-\mathrm{H}), 8.34$ (s, $1 \mathrm{H}$, coumarin $\mathrm{H}), 8.93(\mathrm{~s}, 1 \mathrm{H}, \mathrm{NH}) 9.90(\mathrm{~s}, 1 \mathrm{H}, \mathrm{NH}) \mathrm{ppm} ;{ }^{13} \mathrm{C} \mathrm{NMR}\left(125 \mathrm{MHz}, \mathrm{CDCl}_{3}\right) \delta: 30.5$, $102.9,103.7,110.7,117.1,117.6,120.8,121.3,122.7,125.4,125.8,126.4,127.8,128.0,128.2$, $128.6,130.1,135.7,137.7,137.9,146.6,151.3,160.7,162.4,164.8,168.4$ ppm; MS (ESI): $m / z$ $485(\mathrm{M}+\mathrm{H})^{+}$. Anal. calcd for $\mathrm{C}_{29} \mathrm{H}_{16} \mathrm{~N}_{4} \mathrm{O}_{4}$ : C, 71.90; H, 3.30; N, 11.57\%. Found: C, 71.78; H, $3.24 ; \mathrm{N}, 11.59 \%$.

5,6-Dihydro-2-(2-oxo-2H-chromen-3-yl)-5-(4-N,N-dimethylaminophenyl)indolo $\left[2^{\prime}, 3\right.$ 3':5,6]pyrano[2,3-d]pyrimidin-4(3H)-one (6f). IR $\left(\mathrm{KBr}, \mathrm{cm}^{-1}\right): 3285,1700,1678$, 1667, 1633, 1599, 1208; ${ }^{1} \mathrm{H}$ NMR $\left(500 \mathrm{MHz}, \mathrm{CDCl}_{3}\right) \delta: 2.66\left(\mathrm{~s}, 6 \mathrm{H}, \mathrm{N}\left(\mathrm{CH}_{3}\right)_{2}\right), 5.11(\mathrm{~s}, 1 \mathrm{H}$, $\mathrm{CH}), 6.79-6.93(\mathrm{~m}, 2 \mathrm{H}, \mathrm{Ar}-\mathrm{H}) ; 7.12-7.33(\mathrm{~m}, 4 \mathrm{H}, \mathrm{Ar}-\mathrm{H}) ; 7.48$ (d, 1H, J = 7.6, Ar-H); 7.64 (d, $1 \mathrm{H}, J=8.0, \mathrm{Ar}-\mathrm{H}), 7.86-8.00(\mathrm{~m}, 4 \mathrm{H}, \mathrm{Ar}-\mathrm{H}), 8.29(\mathrm{~s}, 1 \mathrm{H}$, coumarin $\mathrm{H}), 8.84(\mathrm{~s}, 1 \mathrm{H}, \mathrm{NH}) 9.99$ $(\mathrm{s}, 1 \mathrm{H}, \mathrm{NH}) \mathrm{ppm} ;{ }^{13} \mathrm{C} \mathrm{NMR}\left(125 \mathrm{MHz}, \mathrm{CDCl}_{3}\right) \delta: 31.6,43.4,102.5,104.4,111.1,118.1,120.7$, $121.2,123.1,125.0,125.5,126.1,127.6,128.4,128.6,128.9,129.7,135.5,138.0,138.4,147.2$, 150.5, 161.0, 162.2, 165.4, 167.6 ppm; MS(ESI): $m / z 503(\mathrm{M}+\mathrm{H})^{+}$. Anal. calcd for $\mathrm{C}_{30} \mathrm{H}_{22} \mathrm{~N}_{4} \mathrm{O}_{4}$ : C, 71.71; H, 4.38; N, 11.15\%. Found: C, 71.66; H, 4.33; N, 11.12\%.

5,6-Dihydro-2-(2-oxo-2H-chromen-3-yl)-5-(2-nitrophenyl)-indolo [2', 3':5, 6]pyrano[2,3-

d]pyrimidin-4(3H)-one (6g). IR $\left(\mathrm{KBr}, \mathrm{cm}^{-1}\right): 3278,1710,1684,1671,1636,1603,1205 ;{ }^{1} \mathrm{H}$ NMR $\left(500 \mathrm{MHz}, \mathrm{CDCl}_{3}\right) \delta: 5.00(\mathrm{~s}, 1 \mathrm{H}, \mathrm{CH}), 6.83-6.97(\mathrm{~m}, 2 \mathrm{H}, \mathrm{Ar}-\mathrm{H}) ; 7.16-7.39(\mathrm{~m}, 4 \mathrm{H}, \mathrm{Ar}-$ 
H); 7.55 (d, 1H, $J=7.6$, Ar-H); $7.72(\mathrm{~d}, 1 \mathrm{H}, J=8.0$, Ar-H), 7.80-7.94 (m, 4H, Ar-H), 8.34 (s, $1 \mathrm{H}$, coumarin $\mathrm{H}), 8.88(\mathrm{~s}, 1 \mathrm{H}, \mathrm{NH}) 9.94(\mathrm{~s}, 1 \mathrm{H}, \mathrm{NH}) \mathrm{ppm} ;{ }^{13} \mathrm{C} \mathrm{NMR}\left(125 \mathrm{MHz}, \mathrm{CDCl}_{3}\right) \delta: 30.4$, 102.4, 103.8, 110.8, 117.7, 120.3, 121.7, 122.8, 124.9, 125.6, 126.4, 127.6, 128.2, 128.5, 128.7, $130.2,135.3,137.5,137.7,146.6,150.7,160.7,162.6,164.9,168.3$ ppm; MS (ESI): $m / z 505$ $(\mathrm{M}+\mathrm{H})^{+}$. Anal. calcd for $\mathrm{C}_{28} \mathrm{H}_{16} \mathrm{~N}_{4} \mathrm{O}_{6}: \mathrm{C}, 66.66 ; \mathrm{H}, 3.17 ; \mathrm{N}, 11.11 \%$. Found: $\mathrm{C}, 66.56 ; \mathrm{H}, 3.13$; $\mathrm{N}, 11.12 \%$.

5,6-Dihydro-2-(2-oxo-2H-chromen-3-yl)-5-(3-methylphenyl)-indolo[2',3':5,6] pyrano [2,3d]pyrimidin-4(3H)-one (6h). IR $\left(\mathrm{KBr}, \mathrm{cm}^{-1}\right): 3272,1712,1682,1668,1635,1602,1212 ;{ }^{1} \mathrm{H}$ NMR $\left(500 \mathrm{MHz}, \mathrm{CDCl}_{3}\right) \delta: 2.26\left(\mathrm{~s}, 3 \mathrm{H}, \mathrm{CH}_{3}\right), 5.09(\mathrm{~s}, 1 \mathrm{H}, \mathrm{CH}), 6.74-6.97(\mathrm{~m}, 2 \mathrm{H}, \mathrm{Ar}-\mathrm{H})$; 7.20-7.38 (m, 4H, Ar-H); 7.59 (d, 1H, $J=7.6$, Ar-H); 7.74 (d, $1 \mathrm{H}, J=8.0$, Ar-H), 7.87-8.05 (m, $4 \mathrm{H}, \mathrm{Ar}-\mathrm{H}), 8.37(\mathrm{~s}, 1 \mathrm{H}$, coumarin $\mathrm{H}), 8.85(\mathrm{~s}, 1 \mathrm{H}, \mathrm{NH}) 9.90(\mathrm{~s}, 1 \mathrm{H}, \mathrm{NH}) \mathrm{ppm} ;{ }^{13} \mathrm{C} \mathrm{NMR}(125$ $\left.\mathrm{MHz}, \mathrm{CDCl}_{3}\right) \delta: 19.7,31.5,102.3,104.1,111.4,117.5,120.1,121.8,123.3,124.8,125.7,126.6$, 127.3, 128.2, 128.4, 128.6, 129.9, 135.4, 137.4, 137.8, 147.2, 150.6, 161.3, 162.3, 164.8, 167.7 ppm; MS(ESI): $m / z 474(\mathrm{M}+\mathrm{H})^{+}$. Anal. calcd for $\mathrm{C}_{29} \mathrm{H}_{19} \mathrm{~N}_{3} \mathrm{O}_{4}: \mathrm{C}, 73.57 ; \mathrm{H}, 4.01 ; \mathrm{N}, 8.88 \%$. Found: C, 73.44; H, 4.04; N, 8.82\%.

5,6-Dihydro-2-(2-oxo-2H-chromen-3-yl)-5-(4-chlorophenyl)-indolo[2',3':5, 6]pyrano[2,3d]pyrimidin-4(3H)-one (6i). IR $\left(\mathrm{KBr}, \mathrm{cm}^{-1}\right): 3263,1710,1688,1674,1641,1596,1210 ;{ }^{1} \mathrm{H}$ NMR $\left(500 \mathrm{MHz}, \mathrm{CDCl}_{3}\right) \delta: 4.88(\mathrm{~s}, 1 \mathrm{H}, \mathrm{CH}), 6.80-6.90(\mathrm{~m}, 2 \mathrm{H}, \mathrm{Ar}-\mathrm{H}) ; 7.04-7.27(\mathrm{~m}, 4 \mathrm{H}, \mathrm{Ar}-$ $\mathrm{H}) ; 7.55$ (d, $1 \mathrm{H}, J=7.6$, Ar-H); 7.74 (d, $1 \mathrm{H}, J=8.0, \mathrm{Ar}-\mathrm{H}), 7.84-8.03$ (m, 4H, Ar-H), 8.33 (s, $1 \mathrm{H}$, coumarin $\mathrm{H}), 8.77(\mathrm{~s}, 1 \mathrm{H}, \mathrm{NH}) 10.12(\mathrm{~s}, 1 \mathrm{H}, \mathrm{NH}) \mathrm{ppm} ;{ }^{13} \mathrm{C} \mathrm{NMR}\left(125 \mathrm{MHz}, \mathrm{CDCl}_{3}\right) \delta$ : $31.7,102.2$, 104.2, 111.7, 118.5, 120.3, 121.5, 123.3, 125.3, 125.7, 126.5, 127.3, 128.3, 128.7, 129.1, 129.7, 134.9, 138.3, 138.6, 147.2, 151.0, 161.3, 162.5, 164.6, $168.0 \mathrm{ppm}$; MS(ESI): $\mathrm{m} / \mathrm{z}$ $493.4(\mathrm{M}+\mathrm{H})^{+}$. Anal. calcd for $\mathrm{C}_{28} \mathrm{H}_{16} \mathrm{ClN}_{3} \mathrm{O}_{4}$ : C, 68.09; H, 3.24; N, 8.51\%. Found: $\mathrm{C}, 68.05 ; \mathrm{H}$, $3.20 ; \mathrm{N}, 8.49 \%$.

5,6-Dihydro-2-(2-oxo-2H-chromen-3-yl)-5-(4-methylphenyl)-indolo [2',3':5,6]pyrano [2,3d]pyrimidin-4(3H)-one (6j). IR (KBr, cm $\left.{ }^{-1}\right): 3266,1703,1689,1670,1638,1600,1211 ;{ }^{1} \mathrm{H}$ NMR (500 MHz, $\left.\mathrm{CDCl}_{3}\right) \delta: 2.20\left(\mathrm{~s}, 3 \mathrm{H}, \mathrm{CH}_{3}\right), 5.04(\mathrm{~s}, 1 \mathrm{H}, \mathrm{CH}), 6.77-6.99(\mathrm{~m}, 2 \mathrm{H}, \mathrm{Ar}-\mathrm{H})$; 7.22-7.39 (m, 4H, Ar-H); 7.57 (d, 1H, $J=7.6$, Ar-H); 7.77 (d, 1H, J=8.0, Ar-H), 7.90-8.07 (m, $4 \mathrm{H}, \mathrm{Ar}-\mathrm{H}), 8.39(\mathrm{~s}, 1 \mathrm{H}$, coumarin $\mathrm{H}), 8.81(\mathrm{~s}, 1 \mathrm{H}, \mathrm{NH}) 9.90(\mathrm{~s}, 1 \mathrm{H}, \mathrm{NH}) \mathrm{ppm} ;{ }^{13} \mathrm{C} \mathrm{NMR}(125$ $\left.\mathrm{MHz}, \mathrm{CDCl}_{3}\right) \delta: 19.4,31.4,102.6,104.6,111.5,117.3,120.3,121.3,123.7,124.7,125.8,126.8$, $127.5,128.4,128.8,129.4,129.9,135.4,137.4,137.8,147.2,150.3,161.5,162.7,164.8,168.3$ ppm; MS(ESI): $m / z 474(\mathrm{M}+\mathrm{H})^{+}$. Anal. calcd for $\mathrm{C}_{29} \mathrm{H}_{19} \mathrm{~N}_{3} \mathrm{O}_{4}: \mathrm{C}, 73.57 ; \mathrm{H}, 4.01 ; \mathrm{N}, 8.88 \%$. Found: C, 73.49; H, 4.00; N, 8.77\%.

\section{RESULTS AND DISCUSSION}

The aim of this presented protocol is to highlight the development of a new eco-compatible strategy for the synthesis of 5,6-dihydro-2-(2-oxo- $2 H$-chromen-3-yl)-5-phenyl-indolo$\left[2^{\prime}, 3^{\prime}: 5,6\right]$ pyrano $[2,3-d]$ pyrimidin- $4(3 H)$-ones by the condensation reaction of 2-amino-4,5dihydro-4-phenyl-pyrano[3,2-b]indole-3-carbonitrile derivatives with coumarine-3-carboxylic acid using SuSA under solvent-free conditions (Scheme 3).

To find out the suitable conditions for the synthesis of 2-amino-4,5-dihydro-4-phenylpyrano[3,2- $b]$ indole-3-carbonitrile, a series of experiments were performed with the standard reaction of benzaldehyde (1a), 3-hydroxyindole (2), malononitrile (3), as a model reaction (Scheme 1).

Our initial work started with screening of solvent and catalyst loading so as to identify optimal reaction conditions for the synthesis of pyranoindole derivatives. To evaluate the effect of solvent, we studied the reaction of benzaldehyde, 3-hydroxyindole and malononitrile in the presence of catalytic amount of TUD $(0.1 \mathrm{mmol})$. A range of solvents like acetonitrile, 1,4- 
dioxane, $\mathrm{CHCl}_{3}, \mathrm{MeOH}, \mathrm{EtOH}$ and water were examined (Table 1, Entries 1-6). The reaction without any solvent at $70{ }^{\circ} \mathrm{C}$ was not very successful (Table 1, Entry 5). The reaction was more facile and proceeded to give highest yield, in the presence of water as solvent (Table 1, Entry 6). Furthermore, the effect of reaction temperature was examined and the reaction proceeded smoothly at $70{ }^{\circ} \mathrm{C}$ (Table 1, Entry 6). The model reaction was conducted in a range of different temperatures, including room temperature, 50, 60, 70 and $80{ }^{\circ} \mathrm{C}$, in the presence of $0.1 \mathrm{mmol}$ TUD catalyst in water (Table 1, Entries 6-10). As can be concluded from Table 1, the reaction proceeded slowly at room temperature. With increasing temperature to $70^{\circ} \mathrm{C}$, reaction yield was increased and time of reaction was decreased, when the reaction was heated above $70{ }^{\circ} \mathrm{C}$, so high temperatures did not further improved yield and decrease time of reaction. The greatest yield in the shortest reaction time was obtained in water at $70{ }^{\circ} \mathrm{C}$ (Table 1, Entry 6). We also evaluated the amount of TUD required for the reaction. Catalyst loadings in the range of 0.00 $0.15 \mathrm{mmol}$ were tested (Table 1, Entries 6 and 11-14). The best result was obtained with 0.1 mmol of TUD in water at $70{ }^{\circ} \mathrm{C}$ (Table 1, Entry 6). Among the different catalysts tested, including $\mathrm{KF} / \mathrm{Al}_{2} \mathrm{O}_{3}$, triphenyl phosphine $\left(\mathrm{PPh}_{3}\right)$, tetrabutylammonium bromide (TBAB), $p$-toluene sulfonic acid and TUD, TUD was found to be the most efficient in terms of the reaction time and yield of the product (Table 1, Entries 6 and 15-18).

Encouraged by this successful three-component reaction, synthesis of diverse 2-amino-4,5dihydro-4-phenyl-pyrano[3,2-b]indole-3-carbonitrile derivatives $\mathbf{4 a - j}$ were undertaken. The aromatic aldehydes bearing electron-withdrawing and electron donating groups were found to be equally effective to produce 2-amino-4,5-dihydro-4-phenyl-pyrano[3,2- $b$ ] indole-3carbonitrile derivatives 4a-j in very good yields (Table 2, Entries 1-10).

Table 1. Optimization of the reaction conditions on the synthesis of 2-amino-4,5-dihydro-4-phenylpyrano[3,2-b]indole-3-carbonitrile $\mathbf{4} \mathbf{a}^{\mathbf{a}}$.

\begin{tabular}{|c|c|c|c|c|c|c|}
\hline Entry & Catalyst & Amount & Solvent & Temperature $\left({ }^{\circ} \mathrm{C}\right)$ & Time (min) & Yield (\%) $)^{\mathrm{b}}$ \\
\hline 1 & TUD & $0.1 \mathrm{mmol}$ & $\mathrm{CH}_{3} \mathrm{CN}$ & Reflux & 120 & 54 \\
\hline 2 & TUD & $0.1 \mathrm{mmol}$ & $1,4-$ Dioxane & Reflux & 120 & 56 \\
\hline 3 & TUD & $0.1 \mathrm{mmol}$ & $\mathrm{CHCl}_{3}$ & Reflux & 120 & 39 \\
\hline 4 & TUD & $0.1 \mathrm{mmol}$ & $\mathrm{MeOH}$ & Reflux & 90 & 73 \\
\hline 5 & TUD & $0.1 \mathrm{mmol}$ & $\mathrm{EtOH}$ & Reflux & 90 & 78 \\
\hline 6 & TUD & $0.1 \mathrm{mmol}$ & $\mathrm{H}_{2} \mathrm{O}$ & 70 & 40 & 94 \\
\hline 7 & TUD & $0.1 \mathrm{mmol}$ & $\mathrm{H}_{2} \mathrm{O}$ & $\mathrm{rt}$ & 120 & 59 \\
\hline 8 & TUD & $0.1 \mathrm{mmol}$ & $\mathrm{H}_{2} \mathrm{O}$ & 50 & 90 & 69 \\
\hline 9 & TUD & $0.1 \mathrm{mmol}$ & $\mathrm{H}_{2} \mathrm{O}$ & 60 & 60 & 81 \\
\hline 10 & TUD & $0.1 \mathrm{mmol}$ & $\mathrm{H}_{2} \mathrm{O}$ & 80 & 40 & 96 \\
\hline 11 & TUD & $0.0 \mathrm{mmol}$ & $\mathrm{H}_{2} \mathrm{O}$ & 70 & 150 & 25 \\
\hline 12 & TUD & $0.02 \mathrm{mmol}$ & $\mathrm{H}_{2} \mathrm{O}$ & 70 & 90 & 47 \\
\hline 13 & TUD & $0.05 \mathrm{mmol}$ & $\mathrm{H}_{2} \mathrm{O}$ & 70 & 65 & 74 \\
\hline 14 & TUD & $0.15 \mathrm{mmol}$ & $\mathrm{H}_{2} \mathrm{O}$ & 70 & 40 & 94 \\
\hline 15 & KF/Al $\mathrm{O}_{3}$ & $0.1 \mathrm{mmol}$ & $\mathrm{H}_{2} \mathrm{O}$ & 70 & 120 & 66 \\
\hline 16 & PPh & $0.1 \mathrm{mmol}$ & $\mathrm{H}_{2} \mathrm{O}$ & 70 & 90 & 70 \\
\hline 17 & TBAB & $0.1 \mathrm{mmol}$ & $\mathrm{H}_{2} \mathrm{O}$ & 70 & 120 & 79 \\
\hline 18 & $p$-TSA & $0.1 \mathrm{mmol}$ & $\mathrm{H}_{2} \mathrm{O}$ & 70 & 120 & 38 \\
\hline
\end{tabular}

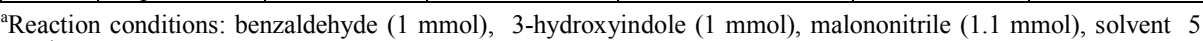
$\mathrm{mL}$. 'Isolated yield.

After the synthesis of 2-amino-4,5-dihydro-4-phenylpyrano[3,2-b]indole-3-carbonitriles, we have synthesized 5,6-dihydro-2-(2-oxo-2H-chromen-3-yl)-5-phenyl-indolo[2',3':5,6]pyrano [2,3-d]pyrimidin-4(3H)-one derivatives.

The solvents played an important role in the synthesis of indolo pyrano pyrimidinone derivatives. Various reaction media were screened (methanol, acetonitrile, $t$ - $\mathrm{BuOH}, 1,4$-dioxane, 
ethanol and THF) using the model reaction (Table 3, Entries 1-6). The reaction was carried out under solvent-free condition at $80^{\circ} \mathrm{C}$. It was found that the best results were obtained with 0.05 mmol of SuSA under solvent free condition (Table 3, Entry 7). The reaction was completed in 3 $\mathrm{h}$ and the expected product was obtained in $90 \%$ yield. Next, the effect of temperature was evaluated for the model reaction. It was observed that the reaction did not proceed at room temperature. Elevating the reaction temperature proved helpful, and the yield of desired product increased considerably (Table 3, Entries 7-12).

Table 2. Synthesis of various 2-amino-4,5-dihydro-4-phenyl-pyrano[3,2-b]indole-3-carbonitrile derivatives from benzaldehyde, 3-hydroxyindole, malononitrile catalysed by TUD $(0.1 \mathrm{mmol})^{\mathrm{a}}$.

\begin{tabular}{|c|c|c|c|c|}
\hline Entry & $\mathrm{R} 1$ & Product & Time (min) & Yield (\%) $^{\mathrm{b}}$ \\
\hline 1 & $\mathrm{H}$ & $\mathbf{4 a}$ & 40 & 94 \\
\hline 2 & $4-\mathrm{F}$ & $\mathbf{4 b}$ & 30 & 88 \\
\hline 3 & $3-\mathrm{Br}$ & $\mathbf{4 c}$ & 30 & 89 \\
\hline 4 & $2-\mathrm{Cl}$ & $\mathbf{4 d}$ & 60 & 84 \\
\hline 5 & $4-\mathrm{CN}$ & $\mathbf{4}$ & 40 & 85 \\
\hline 6 & $4-\mathrm{N}\left(\mathrm{CH}_{3}\right)_{2}$ & $\mathbf{4 f}$ & 60 & 87 \\
\hline 7 & $2-\mathrm{NO}_{2}$ & $\mathbf{4 g}$ & 60 & 85 \\
\hline 8 & $3-\mathrm{CH}_{3}$ & $\mathbf{4 h}$ & 60 & 84 \\
\hline 9 & $4-\mathrm{Cl}_{2}$ & $\mathbf{4 i}$ & 60 & 86 \\
\hline 10 & $4-\mathrm{CH}_{3}$ & $\mathbf{4 j}$ & 60 & 85 \\
\hline
\end{tabular}

${ }^{\text {a }}$ Reaction conditions: benzaldehyde $(1 \mathrm{mmol})$, 3-hydroxyindole $(1 \mathrm{mmol})$, malononitrile $(1.1 \mathrm{mmol})$; TUD $0.1 \mathrm{mmol}$ in water at $70^{\circ} \mathrm{C}$. Isolated yield ${ }^{b}$

Table 3. Optimization of the reaction conditions on the synthesis of 5,6-dihydro-2-(2-oxo- $2 H$-chromen-3yl)-5-phenyl-indolo[2',3':5,6]pyrano[2,3-d]pyrimidin-4(3H)-one $\mathbf{6 a}^{\mathrm{a}}$.

\begin{tabular}{|c|c|c|c|c|c|c|}
\hline Entry & Catalyst & Amount & Solvent & Temperature $\left({ }^{\circ} \mathrm{C}\right)$ & Time $(\mathrm{h})$ & Yield $(\%)^{\mathrm{b}}$ \\
\hline 1 & SuSA & $0.05 \mathrm{mmol}$ & $\mathrm{MeOH}$ & Reflux & 4.0 & 77 \\
\hline 2 & SuSA & $0.05 \mathrm{mmol}$ & $\mathrm{CH}_{3} \mathrm{CN}$ & Reflux & 5.0 & 42 \\
\hline 3 & SuSA & $0.05 \mathrm{mmol}$ & $t$-BuOH & Reflux & 4.0 & 32 \\
\hline 4 & SuSA & $0.05 \mathrm{mmol}$ & $1,4-$ Dioxane & Reflux & 4.0 & 65 \\
\hline 5 & SuSA & $0.05 \mathrm{mmol}$ & EtOH & Reflux & 4.0 & 81 \\
\hline 6 & SuSA & $0.05 \mathrm{mmol}$ & THF & Reflux & 4.0 & 47 \\
\hline 7 & SuSA & $0.05 \mathrm{mmol}$ & Solvent-free & 80 & 3.0 & 90 \\
\hline 8 & SuSA & $0.05 \mathrm{mmol}$ & Solvent-free & Rt & 7.0 & 54 \\
\hline 9 & SuSA & $0.05 \mathrm{mmol}$ & Solvent-free & 50 & 6.0 & 63 \\
\hline 10 & SuSA & $0.05 \mathrm{mmol}$ & Solvent-free & 60 & 5.0 & 73 \\
\hline 11 & SuSA & $0.05 \mathrm{mmol}$ & Solvent-free & 70 & 3.0 & 80 \\
\hline 12 & SuSA & $0.05 \mathrm{mmol}$ & Solvent-free & 90 & 6.0 & 22 \\
\hline 13 & SuSA & $0.00 \mathrm{mmol}$ & Solvent-free & 80 & 5.0 & 64 \\
\hline 14 & SuSA & $0.02 \mathrm{mmol}$ & Solvent-free & 80 & 4.0 & 78 \\
\hline 15 & SuSA & $0.04 \mathrm{mmol}$ & Solvent-free & 80 & 3.0 & 90 \\
\hline 16 & SuSA & $0.06 \mathrm{mmol}$ & Solvent-free & 80 & 4.0 & 68 \\
\hline 17 & MTSA & $0.05 \mathrm{mmol}$ & Solvent-free & 80 & 4.0 & 73 \\
\hline 18 & CSA & $0.05 \mathrm{mmol}$ & Solvent-free & 80 & 4.0 & 76 \\
\hline 19 & SPA & $0.05 \mathrm{mmol}$ & Solvent-free & 80 & 80 & 70 \\
\hline 20 & SA & $0.05 \mathrm{mmol}$ & Solvent-free & 8.0 & \\
\hline
\end{tabular}

${ }^{a}$ Reaction conditions: $4 a(1 \mathrm{mmol})$ and coumarin-3-carboxylic acid $(1 \mathrm{mmol})$, solvent $5 \mathrm{~mL}$. ${ }^{\mathrm{b}}$ Isolated yield.

In order to evaluate the appropriate catalyst loading, the model reaction was performed using 0.0 to $0.06 \mathrm{mmol} \mathrm{SuSA}$ at $80{ }^{\circ} \mathrm{C}$ without solvent (Table 3, Entries 7 and 13-16). It was found that $0.05 \mathrm{mmol}$ of the catalyst afforded the maximum yield in minimum time. Higher 
percentages of catalyst loading $(0.06 \mathrm{mmol})$ neither increased the yield nor lowered the conversion time. To show that SuSA is an efficient catalyst, we accomplished the model reaction in the presence of catalysts, such as melamine trisulfonic acid (MTSA), cellulose sulfuric acid (CSA), silica perchloric acid (SPA) and sulfamic acid (SA) (Table 3, Entries 1720). Tested catalysts gave lower yields compared with SuSA (Table 3, Entry 7). Thus, solventfree and $0.05 \mathrm{mmol}$ of SuSA were chosen as the optimum system to extend the protocol.

At these optimise conditions (solvent-free, $80{ }^{\circ} \mathrm{C}, 0.05 \mathrm{mmol}$ of SuSA) we synthesized various indolo pyrano pyrimidinones 6a-j (Table 4, Entries 1-10). All the synthesized compounds were confirmed by their analytical and spectroscopic data.

Table 4. Preparation of various 5,6-dihydro-2-(2-oxo-2H-chromen-3-yl)-5-phenyl-indolo$\left[2^{\prime}, 3^{\prime}: 5,6\right]$ pyrano[2,3-d]pyrimidin-4(3H)-one derivatives ${ }^{\mathrm{a}}$.

Entry (\%)

Bull. Chem. Soc. Ethiop. 2015, 29(3) 


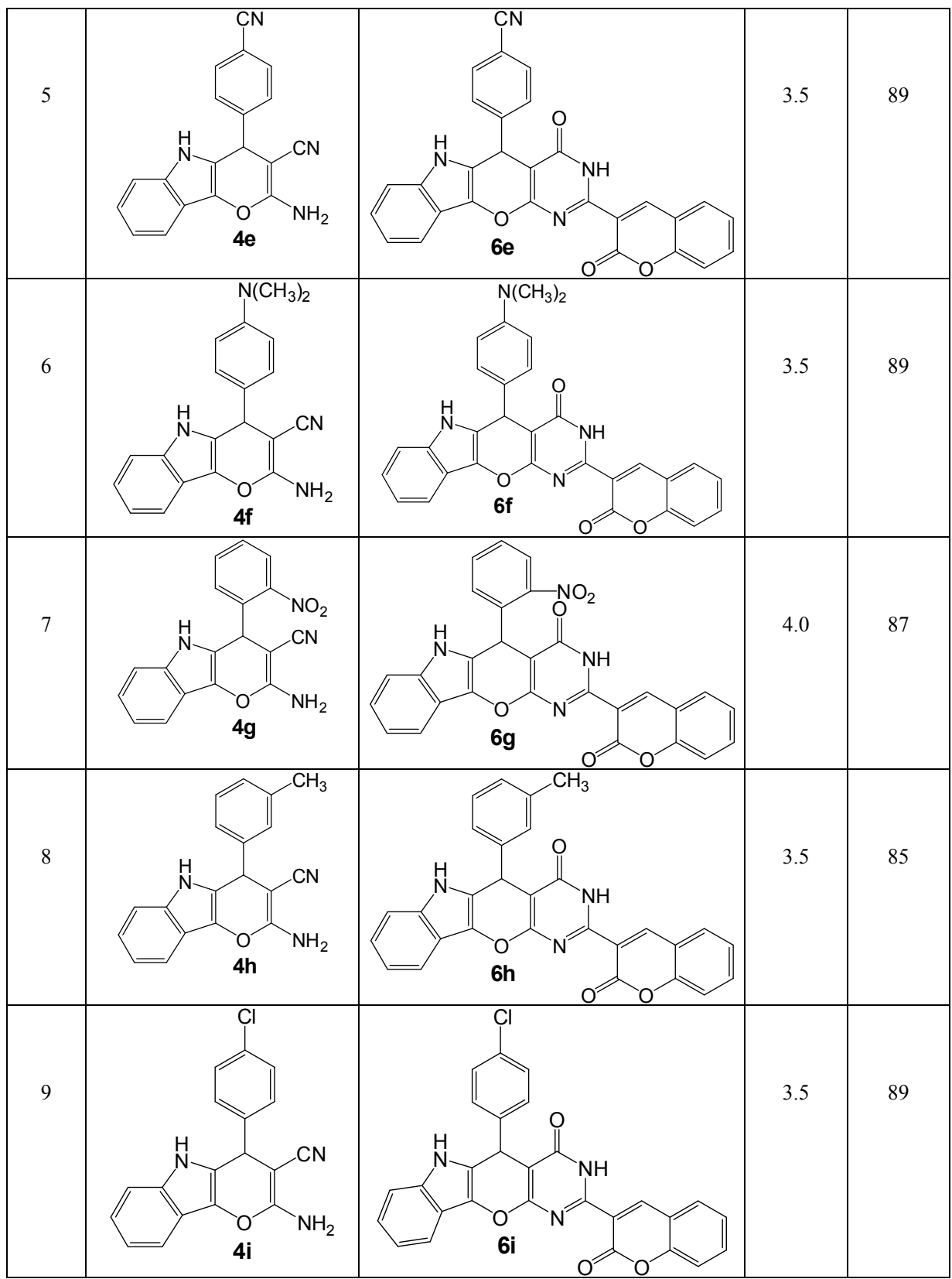

Bull. Chem. Soc. Ethiop. 2015, 29(3) 


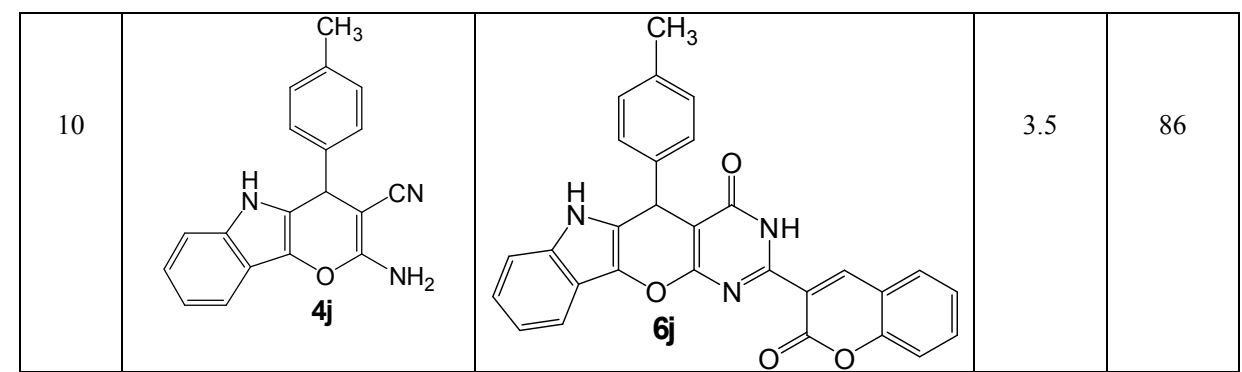

${ }^{\mathrm{a}}$ Reaction conditions: 4a-j $(1 \mathrm{mmol})$ and coumarin-3-carboxylic acid $(1 \mathrm{mmol})$ in the presence of SuSA $(0.05$

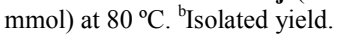

The possible mechanism for the synthesis of 5,6-dihydro-2-(2-oxo-2 $H$-chromen-3-yl)-5phenyl-indolo[2',3':5,6]pyrano[2,3-d]pyrimidin-4(3H)-one derivatives in the presence of SuSA as a solid catalyst is shown in Scheme 4. SuSA activates the coumarin-3-carboxylic acid by protonation to form a cation intermediate (a). In continue, the formation of (b) resulting from the amidation of (a) with $\mathbf{4 a}$ was established. In the next step, the protonation of nitrile group of intermediate (b) following by a cyclo-addition reaction was occurred to form the intermediate (c). In continue the addition reaction of $-\mathrm{SO}_{3}{ }^{-}$followed by ring opening of the (c) to the intermediate (d) and (e) followed by ring closure of intermediate (e) results in the formation of intermediate (f) that convert to the (6a) as product by the de-protonation reaction. Interestingly, the formation of compound $\mathbf{6 a}$, obtained from the condensation of coumarin-3-carboxylic acid with $4 \mathbf{a}$, confirms the mechanism of the reaction which was rarely described in the literature as Dimroth rearrangement $[35,36]$.

The reusability of the catalyst is one of the most important green aspects by avoiding toxic catalyst [37]. One of the most important advantages of heterogeneous catalysis over the homogeneous counterpart is the possibility of reusing the catalyst by simple filtration, without loss of activity [38-40]. We have studied the recyclability and reusability of the catalyst. After completion of the reaction as indicated TLC, the insoluble crude product was dissolved in hot ethanol and the SuSA was filtered off. The filtrate was concentrated to dryness, and the crude product was purified by recrystallization from ethanol. The recovered catalyst was, washed with acetone, dried and reused for subsequent reactions without significant loss in its activity. The catalyst was recycled for four runs without loss of its activity (Figure 1). 


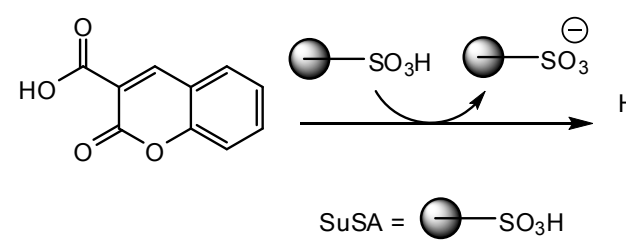<smiles>O=C(O)c1cc2ccccc2oc1=O</smiles><smiles>CC(C)(C)OC(=O)O</smiles>

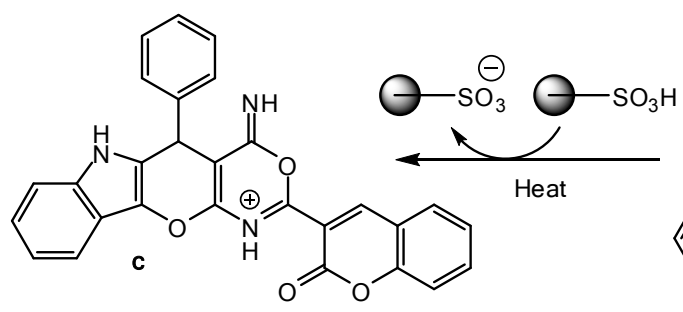<smiles>N#CC1=C(NC(=O)c2cc3ccccc3oc2=O)Oc2c([nH]c3ccccc23)C1c1ccccc1</smiles>

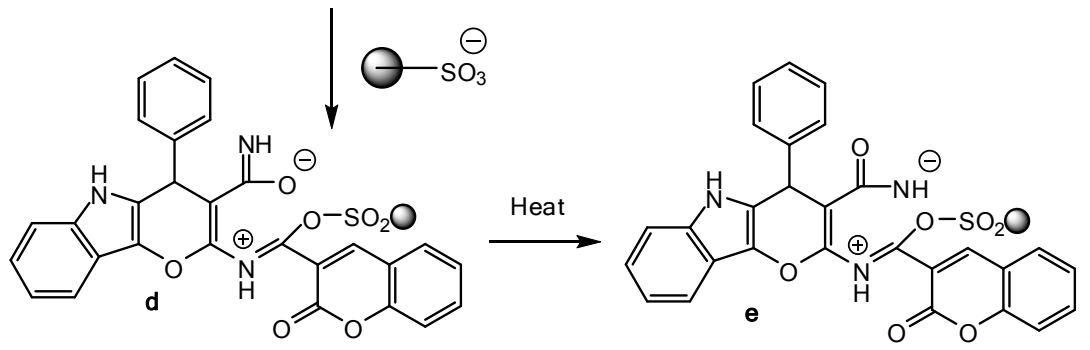

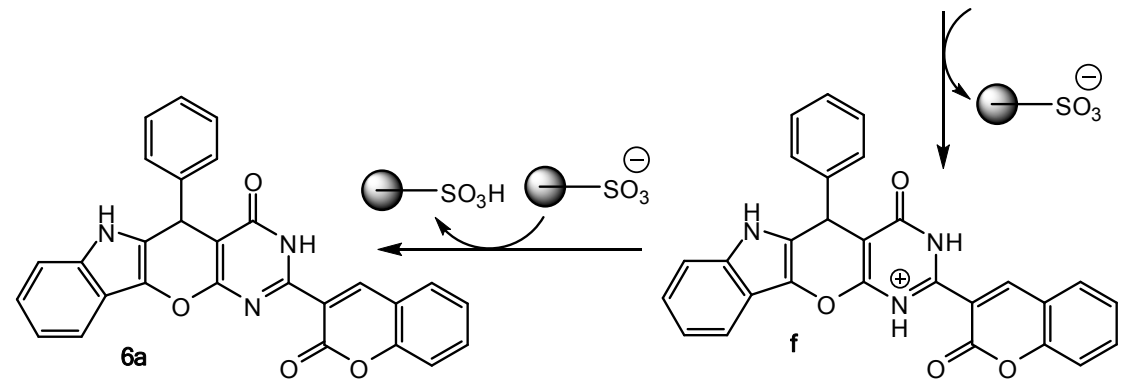

Scheme 4. A possible mechanism for the synthesis of 5,6-dihydro-2-(2-oxo- $2 H$-chromen-3-yl)5-phenyl-indolo[2',3':5,6]pyrano[2,3-d]pyrimidin-4(3H)-one derivatives in the presence of SuSA as a catalyst.

Bull. Chem. Soc. Ethiop. 2015, 29(3) 


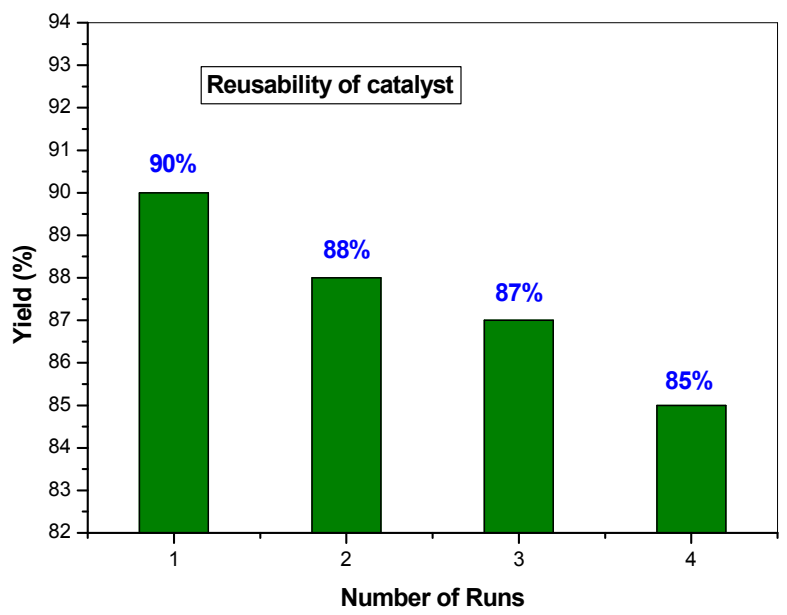

Figure 1. Recyclability of the catalyst: The SuSA catalyst could be reused four times without any loss of its activity towards the synthesis of 5,6-dihydro-2-(2-oxo- $2 \mathrm{H}$-chromen-3yl)-5-phenyl-indolo[2',3':5,6]pyrano[2,3-d]pyrimidin-4(3H)-one.

\section{CONCLUSIONS}

In summary, we have developed a straightforward and efficient method for the preparation of 5,6-dihydro-2-(2-oxo-2 $H$-chromen-3-yl)-5-phenyl-indolo[2',3':5,6]pyrano[2,3- $d]$ pyrimidin-

$4(3 H)$-one derivatives by the condensation of 2-amino-4,5-dihydro-4-phenylpyrano[3,2$b$ ]indole-3-carbonitriles with coumarin-3-carboxylic acid in the presence of $0.05 \mathrm{mmol}$ of SuSA as catalyst. This method tolerates most of the substrates, and the catalyst can be reused at least four times without significant loss of activity.

\section{ACKNOWLEDGEMENTS}

The authors express their gratitude to the management of C. Abdul Hakeem College, Melvisharam - India, for the facilities and support.

\section{REFERENCES}

1. Witaicenis, A.; Seito, L.N.; Chagas, A.S.; Junior, L.D.A.; Luchini, A.C.; Rodrigues-Orsi, P.; Cestari, S.H.; Stasi, L.C.D. Phytomedicine 2014, 21, 240.

2. Irena, K. Curr. Med. Chem. 2000, 5, 29.

3. Xi, G.-L.; Liu, Z.-Q. Eur. J. Med. Chem. 2013, 68, 385.

4. Jashari, A.; Imeri, F.; Ballazhi, L.; Shabani, A.; Mikhova, B.; Drager, G.; Popovski, E.; Huwiler, A. Bioorg. Med. Chem. 2014, 22, 2655.

5. Zhang, W.; Li, Z.; Zhou, M.; Wu, F.; Hou, X,; Luo, H.; Liu, H.; Han, X.; Yan, G.; Ding, Z.; Li, R. Bioorg. Med. Chem. Lett. 2014, 24, 799.

6. Zhao, L.; Yao, Y.; Li, S.; Lv, M.; Chen, H.; Li, H. Bioorg. Med. Chem. Lett. 2014, 24, 900.

7. Kawate, T.; Iwase, N.; Shimizu, M.; Stanley, S.A.; Wellington, S.; Kazyanskaya, E.; Hung, D.T. Bioorg. Med. Chem. Lett. 2013, 23, 6052. 
8. Kumar, K.A. Bioorg. Med. Chem. Lett. 2013, 23, 6406.

9. Asadipour, A.; Alipour, M.; Jafari, M.; Khoobi, M.; Emami, S.; Nadri, H.; Sakhteman, A.; Moradi, A.; Sheibani, V.; Moghadam, F.H.; Shafiee, A.; Foroumadi, A. Eur. J. Med. Chem. 2013, 70, 623 .

10. David, R. Nat. Rev. Mol. Cell Biol. 2011, 12, 767.

11. Kaufman, J.M.; Reginster, J.Y.; Boonen, S.; Brandi, M.L.; Cooper, C.; Dere, W. Bone 2013, 53, 134.

12. Sashidhara, K.V.; Modukuri, R.K.; Choudhary, D.; Rao, K.B.; Kumar, M.; Khedgikar, V.; Trivedi, R. Eur. J. Med. Chem. 2013, 70, 802.

13. Khan, A.T.; Das, D.K.; Islam, K.; Das, P. Tetrahedron Lett. 2012, 53, 6418.

14. Augustine, J.K.; Bombrun, A.; Ramappa, B.; Boodappa, C. Tetrahedron Lett. 2012, 53, 4422.

15. Mokhtary, M.; Najafizadeh, F. C. R. Chimie 2012, 15, 530.

16. Rao, P.; Konda, S.; Iqbal, J.; Oruganti, S. Tetrahedron Lett. 2012, 53, 5314.

17. Banothu, J.; Bavanthula, R. Chin. Chem. Lett. 2012, 23, 1015.

18. Adrom, B.; Hazeri, N.; Maghsoodlou, M.T.; Mollamohammadi, M. Res. Chem. Intermed. 2014, doi: 10.1007/s11164-014-1564-2.

19. Kiyani, H.; Ghiasi, M. Res. Chem. Intermed. 2014, doi: 10.1007/s11164-014-1766-7.

20. Khaligh, N.G.; Mihankhah, T. Res. Chem. Intermed. 2014, doi: 10.1007/s11164-014-15526.

21. Shirini, F.; Khaligh, N.G. Phosphorus, Sulfur Silicon Relat. Elem. 2011, 186, 2156

22. Shirini, F.; Khaligh, N.G. Monatsh. Chem. 2012, 143, 631.

23. Shirini, F.; Khaligh, N.G. Chin. J. Catal. 2013, 34, 695.

24. Hussain, A.M.; Mansoor, S.S.; Aswin, K.; Logaiya, K.; Sudhan, S.P.N. Bull. Chem. Soc. Ethiop. 2014, 28, 91.

25. Mansoor, S.S.; Aswin, K.; Logaiya, K.; Sudhan, S.P.N. J. King Saudi University - Sci. 2013, 25, 191.

26. Mansoor, S.S.; Logaiya, K.; Aswin, K.; Sudhan, S.P.N. J. Taibah Univ. Sci. 2015, 9, 213.

27. Kumar, S.; Jain, S.L.; Sain, B. RSC Adv. 2012, $2,789$.

28. Verma, S.; Jain, S.L. Tetrahedron Lett. 2012, 53, 6055.

29. Verma, S.; Kumar, S.; Jain, S.L. Sain, B. Org. Biomol. Chem. 2011, 9, 6943.

30. Verma, S.; Jain, S.L.; Sain, B. Tetrahedron Lett. 2010, 51, 6897.

31. Verma, S.; Singh, R.; Tripathi, D.; Gupta, P.; Bahuguna, G.M.; Jain, S.L. RSC Adv. 2013, 3, 4184.

32. Mosaddegh, E.; Hassankhani, A. Bull. Chem. Soc. Ethiop. 2012, 26, 461.

33. Karimi-Jaberi, Z.; Fakhraei, H. Bull. Chem. Soc. Ethiop. 2012, 26, 473.

34. Wang, M.; Liu, Y.; Song, Z.; Zhao, S. Bull. Chem. Soc. Ethiop. 2013, 27, 421.

35. Foucourt, A.; Dubouilh-Benard, C.; Chosson, E.; Corbiere, C.; Buquet, C.; Iannelli, M.; Leblond, B.; Marsais, F.; Besson, T. Tetrahedron 2010, 66, 4495.

36. Dimroth, O. Justus Liebigs Ann. Chem. 1909, 364, 183.

37. Patra, A.; Mahapatra, T. J. Indian Chem. Soc. 2012, 89, 925.

38. Teimouri, A.; Ghorbanian, L.; Moatari, A. Bull. Chem. Soc. Ethiop. 2013, 27, 427.

39. Baghernejad, B.; Heravi, M.M.; Oskooie, H.A.; Bamoharram, F.F. Bull. Chem. Soc. Ethiop. 2012, 26, 145.

40. Hakimi, F.; Hassanabadi, A.; Tabatabaee, M.; Heravi, M.M. Bull. Chem. Soc. Ethiop. 2014, 28,67 . 